\title{
Sistema arbolado nativo y carbono en el Cantón Palenque, Provincia de los Ríos, Ecuador
}

\author{
Native wooded system and carbon in the Canton Palenque, Province of \\ los Ríos, Ecuador
}

\section{Patricia Mero ${ }^{1^{*}} \&$ Carmita Bonifaz ${ }^{2}$}

\author{
${ }^{1}$ Empresas Tropack, Analista de laboratorio, Km 26 vía Boliche \\ ${ }^{2}$ Universidad de Guayaquil, Herbario GUAY, Facultad de Ciencias Naturales, Av. Raúl \\ Gómez Lince s.n. y Av. Juan Tanca Marengo (campus Mapasingue), Guayaquil-Ecuador
}

Recibido 10 de abril 2021; recibido en forma revisada 2 de mayo 2021, aceptado 4 de junio 2021

Disponible en línea 26 de junio 2021

\begin{abstract}
Resumen
El presente estudio tuvo como objetivo evaluar el carbono en un sistema arbolado nativo del Cantón Palenque, Provincia de los Ríos, Ecuador; donde se establecieron dos cuadrantes al azar, de $50 \times 50 \mathrm{~m}^{2}$ en el interior del bosque nativo de la Estación Científica Pedro Franco Dávila, se realizaron mediciones dasométricas que fueron tomadas en el campo de estudio como diámetro a la altura del pecho (DAP), altura total $(\mathrm{H})$ y volumen comercial $(\mathrm{Vc})$, donde se empleó el método directo o destructivo para tener las muestra de tronco, fuste, rama, raíces, hojas y hojarasca que fueron enviadas al laboratorio para obtener carbono (c) mediante el método de análisis elemental. Registrando en el cuadrante A1 125 individuos, 12 especies, 12 género y 9 familia cuadrante A2 135 individuos, 12 especies, 12 género y 8 familia; en ambos cuadrante la familia más representativa fue Morácea, y la especie Tillo prieto (Sorocea sarcocarpa) tuvo mayor presencia en ambos cuadrantes; biomasa con un total para el área estudiada de 30,17 T(c), volumen de rama $3.65 \mathrm{~m}^{3}$, volumen comercial de un individuo es de $4.8 \mathrm{~m}^{3}$ volumen de raíces $1.63 \mathrm{~m}^{3}$, hojas 266,6 T (c), hojarasca 634,6 T (c), dando un total en el área muestral con 46 individuos de Tillo prieto (Sorocea sarcocarpa) de 31,09 T(c). En conclusión Tillo prieto (Sorocea sarcocarpa), constituye una población de árboles jóvenes por tener 11 individuos en clase de altura entre 7-13,99 en el cuadrante A1, con siete individuos en clase de altura entre 6- 8,99 en el cuadrante A2.

Palabras claves: Bosque nativo, Carbono, Sorocea sarcocarpa, sistema arbolado, mediciones dasométricas.
\end{abstract}

\begin{abstract}
The present study aimed to evaluate carbon in a native wooded system of the Palenque Canton, Los Ríos Province, Ecuador; where two quadrants were established at random, $50 \times 50 \mathrm{~m} 2$ inside the native forest of the Pedro Franco Dávila Scientific Station, dasometric measurements were made that were taken in the study field as diameter at chest height $(\mathrm{DBH})$, height total $(\mathrm{H})$ and commercial volume $(\mathrm{Vc})$, where the direct or destructive method was used to have the trunk, stem, branch, roots, leaves and leaf litter samples that were sent to the laboratory to obtain carbon (c) by the method of elemental analysis. Registering in quadrant A1 125 individuals, 12 species, 12 genus and 9 family quadrant A2 135 individuals, 12 species, 12 genus and 8 family; In both quadrants, the most representative family was Morácea, and the Tillo prieto species (Sorocea sarcocarpa) had a greater presence in both quadrants; biomass with a total for the studied area of $30.17 \mathrm{~T}$ (c), branch volume $3.65 \mathrm{~m} 3$, commercial volume of an individual is $4.8 \mathrm{~m} 3$ root volume $1.63 \mathrm{~m} 3$, leaves $266.6 \mathrm{~T}$ (c), litter 634, $6 \mathrm{~T}$ (c), giving a total in the sample area with 46 individuals of Tillo prieto (Sorocea sarcocarpa) of $31.09 \mathrm{~T}$ (c). In conclusion, Tillo prieto (Sorocea sarcocarpa), constitutes a population of young trees by having 11 individuals in the height class between 7-13.99 in quadrant $\mathrm{A} 1$, with seven individuals in the height class between 6-8.99 in the quadrant $\mathrm{A} 2$.
\end{abstract}

Keywords: Native forest, Carbon, Sorocea sarcocarpa, wooded system, dasometric measurements.

\footnotetext{
* Correspondencia del autor:

E-mail: paty-lore@hotmail.com - carmita.bonifazb@ug.edu.ec
} 


\section{Introducción}

Los bosques nativos tienen la capacidad de almacenar el carbono presente en la atmosfera, siendo participe del ciclo de este elemento, a los cuales se considera como uno de los principales almacenadores a nivel mundial, debido a los procesos de fotosíntesis y respiración que realizan, en donde la biomasa se concentra en estructuras vegetales como los fustes, ramas, hojas, raíces y cualquier otro tipo de materia orgánica presente (Martel, 2012).

Además, estos sistemas poseen una diversidad biológica muy elevada, compuesta de flora y fauna, las cuales interactúan entre sí, sin embargo, estas zonas están siendo perturbadas por actividades antropogénicas y la tala ilegal, destruyendo así los bosques ecuatorianos (Fernandez, Delgado, Bellet, \& Garcia, 2011). Por lo antes expuesto, es importante evaluar estas especies que brindan conocimientos para cualquier tipo de incentivo económico que puedan ser apoyados por proyectos verdes obteniendo servicio para el autor en estos sistemas presentes (IPCC, 2000).

Para poder cuantificar la cantidad de carbono en las plantas, se aplican diferentes métodos, entre ellos la observación directa o indirecta, por otro lado, también tenemos la metodología propuesta por (Soares \& Oliveira, 2000; Francis, 2000; Gifford, 2000), donde el valor de la cantidad de carbono será desigual según su floema secundario, su epidermis y el crecimiento del árbol, de este modo, el porcentaje de carbono acumulado que está presente en la aérea de la materia orgánica, que a su vez estima el extracto seco sin importa la desigualdad de individuos (Brown \& Lugo, 1984; IPCC, 1996).

Según la investigación de la FAO (2006), los bosques nativos acumulan un billón de toneladas de carbono al año. Siendo la materia seca la responsable de almacenar un $50 \%$. De hecho, existen varios autores donde mencionan que durante el crecimiento los árboles capturan carbono y que a su vez cuando termina su ciclo la misma cantidad que retuvo la retorna a la atmosfera (Lee Pinto, 2002). Así mismo, la captura y almacenamiento del carbono dependerá de su desarrollo (IPPCC, 2007).

Entre los tipos de bosque más importantes del Ecuador, se destacan los siguientes: húmedos, secos, templados, alto andino, nublados y tropicales. Ecuador se encuentra entre los países de Latinoamérica con mayor biodiversidad gracias a tres factores, como lo son: la influencia de las corrientes marinas, su ubicación geográfica en la zona ecuatorial y el levantamiento de las cordilleras de los Andes (Fernandez, Delgado, Bellet, \& Garcia, 2011).

La Estación Científica Pedro Franco Dávila ubicada en la provincia de los Ríos en el cantón Palenque, tiene un bosque protector con una cantidad considerable de arbóreos nativos con estructura leñosa, la cual tiene alterada su capacidad de acumulación de carbono debido a esto causa daño a su contextura vertical y horizontal por ese motivo el bosque se encuentra en reforestación (Bajaña, 2016).

El cuantificar la cantidad de carbono que capturan los árboles nativos, permitirá conocer el rol de estos en el ecosistema del bosque húmedo tropical, el bosque protector denominado Jauneche situado en Estación Científica Pedro Franco Dávila cumple con estas condiciones, de allí el propósito del presente trabajo.

\section{Antecedentes}

La estimación de carbono almacenado en la biomasa aérea en otros países se ha realizado mediante métodos directos e indirectos, como el que se llevó a cabo en la provincia de Santiago del Estero, Argentina, en árboles maderables, encontrándose que dos de ellos presentaron mayor cantidad de carbono así: Quebracho colorado con $10.486 \mathrm{Tn} /$ ha y Quebrachos blanco con 12.697 Tn/ha (Veracruzana, 2009).

En nuestro país, se ha evaluado el carbono almacenado en árboles en diferentes ecosistemas, así Castillo (2014), quien trabajó en el bosque húmedo Pre Montano de "El Prado" en la Provincia de El Oro utilizando el método directo, donde realizaron 10 parcelas al azar en 10 hectáreas identificando un total de 776 individuos, 56 especies y 24 familia estimando el carbono total almacenado de raíz, tallo, ramas y hojas de la sietes especies más abundantes que fueron: Fernán sanchez (Triplaris cumingiana) con 29.14 t/ha, Pacay blanco (Cupania cinérea) con $30.45 \mathrm{t} / \mathrm{ha}$, Laurel (Cordia alliodora) con $19.95 \mathrm{t} /$ ha, Tillo, clavo (Pseudolmedia rigida) con $6.31 \mathrm{t} / \mathrm{ha}$, Membrillo (Inga oerstediana) con $18.85 \mathrm{t} / \mathrm{ha}$, Guaba de mono (Eschweilera integrifolia) con $20.57 \mathrm{t} / \mathrm{ha}$, Vara blanca (Allophylus psilospermus) con $4.87 \mathrm{t} / \mathrm{ha}$.

Arevalo (2015), quien evaluó el almacenamiento de carbono en el bosque natural Tinajillas-Limon Indanza utilizando el método indirecto en cuatro parcelas de un área de 118 hectáreas, se identificaron 925 individuos, 27 especies, 27 géneros y 22 familias. Donde el almacenamiento de carbono en el estrato arbóreo es de 0,064 t con una desviación de 0,114 por el total de individuos presente, y la familia más representativa es Malastomatácea.

Ramirez \& Zamora (2019), evaluaron la biomasa aérea del Bosque Nativo de la Comunidad Rancho Chico de la Provincia Imbabura, utilizando el método indirecto, se identificó en un área de 7.29 ha un total de 396 individuos, 20 especies, 16 géneros y 16 familia. La cantidad de carbono total almacenada fue de 1011.18 ton. En donde las familias: Solanaceae, Actinidiaceae, Myrtaceae, Rubiaceae y Araliaceae contiene más del $50 \%$ de carbono registrando, el género Persea sp con un 0.25 ton. 
Bajaña (2016), realizó un estudio en el bosque protector Pedro Franco Dávila de la provincia de los Ríos utilizando el método indirecto donde se realizó dos parcelas en áreas natural e intervenidas dividida cada una en 4 subparcela de $20 \times 20 \mathrm{~m}$, como resultado para ambas parcelas, la familia más representativa fue Moraceae, del área natural la especie con mayor almacenamiento de carbono fue Tutumbe (Enterolobium cyclocarpum) con 50066 kg ha-1 y del área intervenida fue Fernán Sánchez (Triplaris cumingiana) con 29219 kg ha-1.

\section{Materiales y métodos}

\section{Área de estudio}

Esta investigación se llevó a cabo en la Estación Científica Pedro Franco Dávila en la provincia de los Ríos en el cantón Palenque, Ecuador, la misma que pertenece a la Universidad de Guayaquil de la Facultad de Ciencias Naturales. Los cuadrantes de muestreo se localizaron al interior de bosque nativo en un área secundaria durante los meses de enero y febrero del 2011 en época lluviosa, y para graficar los puntos se utilizaron los programas Google Earth (Google Earth, 2018) y QGIS versión 2.18 (QGIS Development Team 2016) (Figura 1).

\section{Metodología}

\section{Fase de campo}

Especies arbóreas presentes en el sistema arbolado nativo.

Para identificar las especies de un bosque nativo se establecieron dos cuadrantes al azar, de $50 \times 50 \mathrm{~m}$ en el interior del bosque de la Estación Pedro Franco Dávila, con un GPS Garmin y piolas de nylon A-12, dando un área de muestreo total de 5000m2 (Anexo 1).

El cuadrante A1 con las siguientes coordenadas (UTM 648578.68E- 9862757.133N) y una elevación de 60,8275 , exhibió vegetación secundaria con árboles juveniles, encontrándose algunos frutos de piñuelo (Duguetia peruviana) en el suelo El cuadrante A2 con un declive aproximado de $30^{\circ}$, y con las coordenadas (UTM 648433.382E- 9862931.472N) y una elevación de $62,4175 \mathrm{~m}$, encontrándose en el suelo algunos frutos de jaboncillo (Sapindus saponaria L.) y una flor del Tillo Prieto (Sorocea sarcocarpa) (Anexo 2).

En ambos cuadrantes se procedió a etiquetar cada árbol utilizando unas fichas plásticas, las cuales se amarro con hilo de nylon para posteriormente numerarlas. A cada árbol etiquetado se le registro las medidas dasométricas como el diámetro a la altura de pecho (DAP) utilizando una cinta diamétrica y altura total desde la base de suelo hasta el ápice de cada especie. Luego se realizó la identificación de cada árbol presente en cada cuadrante observando las características morfológicas externa del tronco, hojas, flores o frutos además de su nombre común. Todas las características de campo anotadas fueron validadas con la respectiva flora de Jauneche al fin de obtener la familia y el nombre científico (Figura 2).

En cada cuadrante, se eligió un árbol representativo en función a su abundancia, para ser procesado por el método directo o destructivo que requiere de cortar

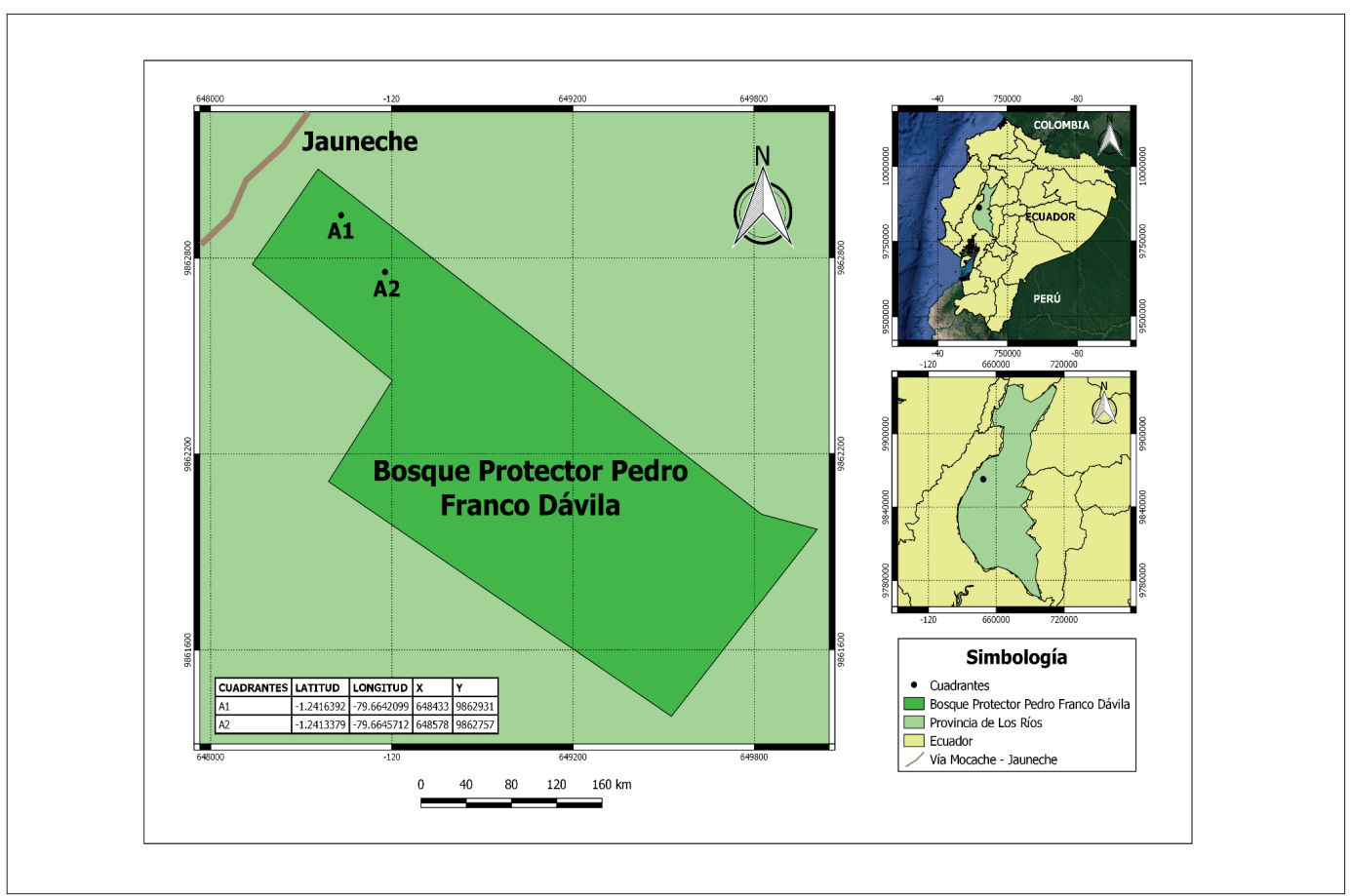

Figura 1. Localización de los cuadrantes en bosque nativo en la Estación científica Pedro Franco Dávila. 


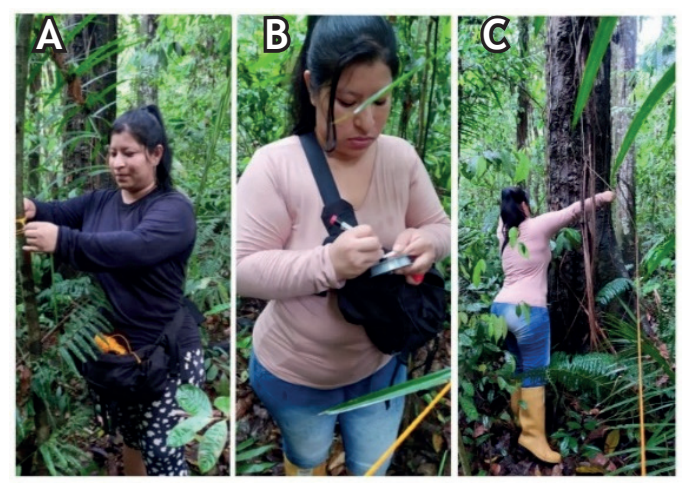

Figura 2. A.-Demarcación de los cuadrantes en bosque secundario. B.-Censo e identificación de las especies. C.Medida del DAP y altura total de la especie.

el árbol para separar muestras de fuste, raíz, hojas, ramas y hojarasca. Las muestras fueron secadas a la sombra y puesta en fundas plásticas para diferenciar las partes recogidas (Figura 3)

\section{Fase de laboratorio}

El árbol escogido para el análisis de carbono fue en función de la especie más abundante, presente en ambas parcelas, siendo el Tillo prieto (Sorocea sarcocarpa). Del individuo apeado con la ayuda de una sierra eléctrica se separó $5 \mathrm{~cm}$ de tronco, 20 $\mathrm{cm}$ de raíz, $20 \mathrm{~cm}$ de rama, un gramo de hojarasca y cinco hojas de una rama de Tillo prieto la misma que después de ser secada a la sombra, se procedió a ponerlas en funda de papel Kraft, etiquetadas con los siguientes códigos: raíz (Pm-R), tronco (Pm-Tr), rama $(\mathrm{Pm}-\mathrm{Rm})$, hoja $(\mathrm{Pm}-\mathrm{H})$, hojarasca $(\mathrm{Pm}-\mathrm{Hr})$ para enviarlas al laboratorio de investigación y vinculación de la Universidad Estatal de Bolívar (ECUMASA) para la determinación de carbono total mediante la prueba de análisis elemental (Figura 4).

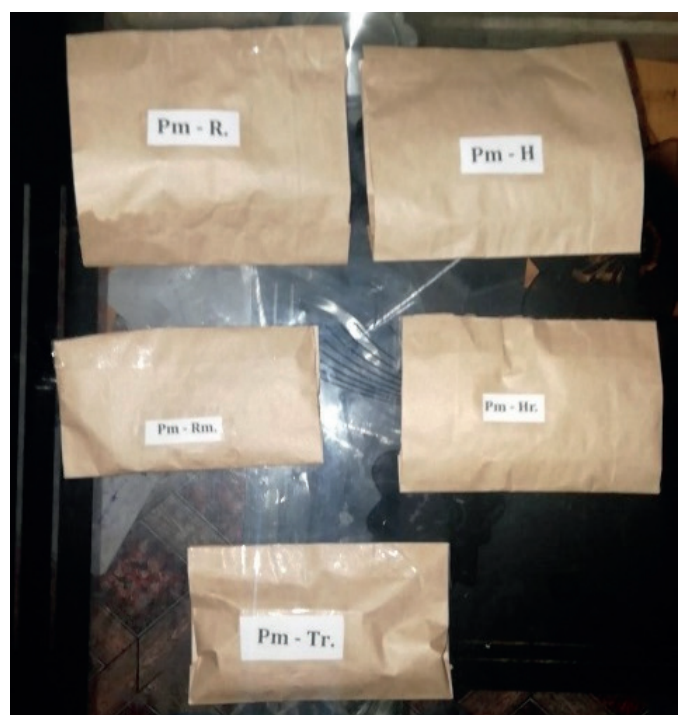

Figura 4. Muestra codificada para el análisis elemental del Tillo prieto (Sorocea sarcocarpa)

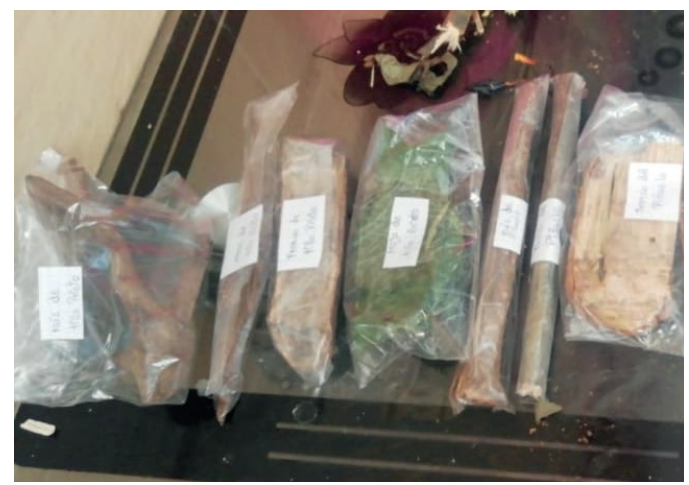

Figura 3. Muestras en fundas plásticas de las partes del árbol seleccionado para análisis de carbono.

\section{Cálculo de carbono}

Para calcular el carbono, se realizó las siguiente formula donde el peso del árbol escogido se multiplicará por el porcentaje de cada muestra que se envió al laboratorio por medio del método de análisis elemental y se dividirá para 100, para posteriormente cuantificar el carbono (Rugnitz, Chacon, \& Porro, 2009).

$$
C=\frac{P a \cdot P}{100}
$$

Dónde:

$$
\begin{aligned}
& \mathrm{C}=\text { Carbono (toneladas) } \\
& \mathrm{Pa}=\text { Peso de árbol } \\
& \mathbf{P}=\text { Porcentaje }
\end{aligned}
$$

\section{Cálculo de la biomasa}

Para calcular la biomasa viva (fuste y copa), mediante las medidas que se tomó en el campo como el diámetro a la altura de pecho (DAP) y altura total del árbol se escogió la categoría de altura, donde se aplicó la fórmula de carbono en toneladas, para posteriormente obtener la biomasa en carbono del cuadrante $\mathrm{A} 1$ y $\mathrm{A} 2$.

\section{Volumen de ramas}

Se procedió mediante el método destructivo del árbol más representativo, a medir todas las ramas de la especie, donde se utilizará la longitud de las ramas lo cual se expresó en metros mediante la siguiente formula

$$
\operatorname{Vr}=(\pi)(r 2)(h)
$$

Donde:

$\mathrm{Vr}=$ volumen de ramas $\left(\mathrm{m}^{3}\right)$

$\mathbf{h}=$ longitud de ramas.

$\mathrm{r}=$ radio de las ramas. 


\section{Volumen comercial}

Se procedió mediante el método destructivo del árbol más representativo, a medir el fuste de la especie lo cual se expresó en la siguiente formula.

$$
\mathrm{VC}=\mathrm{H} . \mathrm{AB} \quad A B=\frac{\mathrm{D} \cdot \mathrm{H}}{\text { Area }}
$$

Donde:

Vc $=$ volumen comercial $\left(\mathrm{m}^{3}\right)$

$\mathrm{h}=$ altura comercial

$\mathrm{AB}=$ Área basal

D= Diámetro.

\section{Volumen raíces}

Se procedió a inferir un tamaño aproximado de las raíces en función de la proyección de la copa del árbol sobre el suelo, se utilizará la longitud de las raíces lo cual se expresará en metros mediante la siguiente fórmula.

$$
\mathrm{Vr}=(\pi)(\mathrm{r} 2)(\mathrm{h})
$$

Donde:

$\mathrm{Vr}=$ volumen de raíces $\left(\mathrm{m}^{3}\right)$

$\mathrm{h}=$ longitud de raíces.

$\mathrm{r}=$ radio de las raíces.

Hojarasca

Para el cálculo de la hojarasca se tomó de 4 punto donde se rastrillo y dio un total de libra y media de hojarasca lo cual solo 10 gramo se utilizó para la lectura del porcentaje de carbono.

\section{Hojas}

Para el cálculo de las hojas se procedió a tomar de la rama la muestra donde dio un total de 3 libras, lo cual solo se utilizó 10 gramos para la lectura del porcentaje de carbono.

\section{Resultados}

Especies arbóreas presentes en el sistema arbolado nativo.

En $5000 \mathrm{~m}^{2}$ de un bosque nativo de la Estación Pedro Franco Dávila se registraron un total de 260 individuos arbóreos con: 9 familias, 13 géneros y 13 especies, con presencia de frutos en el suelo de jaboncillo (Sapindus saponaria L.), Piñuelo (Duguetia peruviana) y una flor en Tillo Prieto (Sorocea sarcocarpa) (Anexo 2).

En el cuadrante $\mathrm{A} 1$ se registró 125 individuos, 9 familias, 12 géneros y 12 especies donde Tillo prieto (Sorocea sarcocarpa) tiene una mayor presencia, con un porcentaje de $20.8 \%$, seguido de la especie Chala (Erythrina sp) y Bantano (Pithecellobium macradenium) ambas con el mismo porcentaje de $15,2 \%$ y Piñuelo (Duguetia peruviana) con un porcentaje de 11,2 (Tabla 1).

La especie con mayor DAP fue: Bantano (Pithecellobium macradenium) con diámetros entre 70 a $79 \mathrm{~cm}$, aunque con pocos individuos que constituyen $0,8 \%$. Las especies con menor DAP de $9 \mathrm{~cm}$ fueron: Coquito (Adelia triloba), Caracolí (Anacardium excelsum), Caucho (Castilla elástica), Moral Bobo (Clarisia racemosa), Piñuelo (Duguetia peruviana), Chala (Erythrina sp), Guayabo de monte (Psidium guajava L), Jaboncillo (Sapindus saponaria $L$ ), Tillo prieto (Sorocea sarcocarpa), Fernán sanchez (Triplaris cumingiana), Tachuelo (Zanthoxylum aff.

\begin{tabular}{|c|c|c|c|c|}
\hline $\begin{array}{l}\text { Nombre } \\
\text { científico }\end{array}$ & Familia & $\begin{array}{l}\text { Nombre } \\
\text { común }\end{array}$ & $\begin{array}{c}\mathrm{N} .^{\circ} \text { de } \\
\text { individuo }\end{array}$ & $\%$ \\
\hline Sorocea sarcocarpa & Moraceae & Tillo prieto & 26 & 20,8 \\
\hline Erythrina sp. & Fabaceae & Chala & 19 & 15,2 \\
\hline Anacardium excelsum & Anacardiaceae & Caracolí & 15 & 2,4 \\
\hline Pithecellobium macradenium & Fabaceae & Bantano & 19 & 15,2 \\
\hline Duguetia peruviana & Annonaceae & Piñuelo & 14 & 11,2 \\
\hline Psidium guajava $L$. & Myrtaceae & Guayabo de monte & 8 & 6,4 \\
\hline Sapindus saponaria L. & Sapindaceae & Jaboncillo & 3 & 2,4 \\
\hline Adelia triloba & Euphorbiaceae & Coquito & 3 & 12 \\
\hline Castilla elástica & Moraceae & Caucho & 8 & 6,4 \\
\hline Zanthoxylum aff. Panamense & Rutaceae & Tachuelo & 3 & 2.4 \\
\hline Triplaris cumingiana & Polygonaceae & Fernán Sánchez & 2 & 1,6 \\
\hline Clarisia racemosa & Moraceae & Moral Bobo & 5 & 4 \\
\hline
\end{tabular}

Tabla 1. Especies arbóreas presentes en el A1 en el bosque nativo de la Estación Pedro Franco Dávila. 
Tabla 2. Número de árboles por clase diamétrica del cuadrante A1 en el bosque nativo de la Estación Pedro Franco Dávila.

\begin{tabular}{ccc}
\hline Clase diamétrica & $\begin{array}{c}\text { N. }{ }^{\circ} \text { de } \\
\text { individuo }\end{array}$ & $\%$ \\
\hline $0-9,99$ & 82 & 65,6 \\
$10-19,99$ & 19 & 15,2 \\
$20-29,99$ & 11 & 8,8 \\
$30-39,99$ & 2 & 1,6 \\
$40-49,99$ & 2 & 1,6 \\
$50-59,99$ & 6 & 4,8 \\
$60-69,99$ & 2 & 1,6 \\
$70-79,99$ & 1 & 0,8 \\
\hline Total & 125 & 100 \\
\hline
\end{tabular}

Panamense) con un mayor número de individuos en un porcentaje de 65,5\% (Tabla 2) (Figura 5).

La especie con mayor altura fue: Caracolí (Anacardium excelsum) con altura ente 40 a $49 \mathrm{~m}$, aunque con pocos individuos que constituyen $1,6 \%$. Las especies con menor altura de $9 \mathrm{~m}$ fueron: Coquito (Adelia triloba), Caucho (Castilla elástica), Moral Bobo (Clarisia racemosa), Piñuelo (Duguetia peruviana), Chala (Erythrina sp), Bantano (Pithecellobium macradenium), Guayabo de monte, (Psidium guajava $L$ ), Jaboncillo (Sapindus saponaria L), Tillo prieto (Sorocea sarcocarpa), Fernán sanchez (Triplaris cumingiana), Tachuelo (Zanthoxylum aff. Panamense) con un mayor número de individuos en un porcentaje de 77,6\% (Tabla 3) (Figura 6).

En el cuadrante A2 se registró 135 individuos, 8 familias, 12 géneros y 12 especies donde Piñuelo (Duguetia peruviana) tiene una mayor presencia con un porcentaje de $21,4 \%$, seguido de la especie Tillo prieto (Sorocea sarcocarpa) con 14,8\% y Caracolí (Anacardium excelsum) con porcentaje de 11,8 (Tabla 4).

Tabla 3. Número de árboles por clase altura del cuadrante A1 en el bosque nativo de la Estación Pedro Franco Dávila.

\begin{tabular}{ccc}
$\begin{array}{c}\text { Clase } \\
\text { altura }\end{array}$ & $\begin{array}{c}\text { N. }{ }^{\circ} \text { de } \\
\text { individuo }\end{array}$ & $\%$ \\
\hline $0-9,99$ & 97 & 77,6 \\
$10-19,99$ & 14 & 11,2 \\
$20-29,99$ & 4 & 3,2 \\
$30-39,99$ & 8 & 6,4 \\
$40-49,99$ & 2 & 1,6 \\
\hline Total & 125 & 100 \\
\hline
\end{tabular}

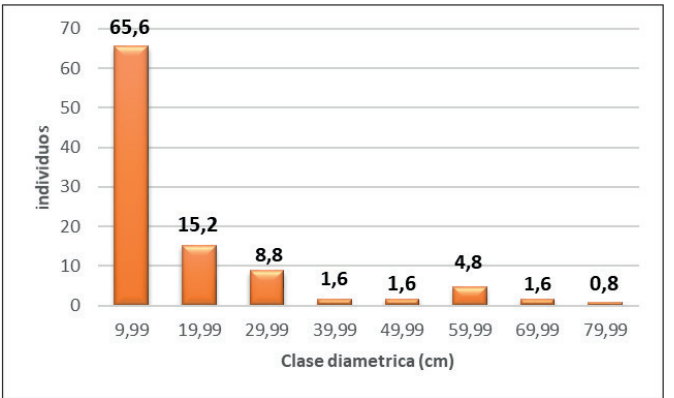

Figura 5. Representación de árboles por clase diamétrica del cuadrante $\mathrm{A} 1$ en el bosque nativo de la Estación Pedro Franco Dávila.

Las especies con mayor DAP fueron: Moral Bobo (Clarisia racemosa), Jobo (Spondias mombin $L$ ) con diámetros entre 42 a $48 \mathrm{~cm}$, aunque con pocos individuos que constituyen 1,4\%. Las especies con menor DAP de $6 \mathrm{~cm}$ fueron: Coquito (Adelia triloba), Caracolí (Anacardium excelsum), Caucho (Castilla elástica), Piñuelo (Duguetia peruviana), Chala (Erythrina sp), Bantano (Pithecellobium macradenium), Guayabo de monte (Psidium guajava $L$ ), Jaboncillo (Sapindus saponaria $L$ ), Tillo prieto (Sorocea sarcocarpa), con un mayor número de individuos en un porcentaje de 46,6\% (Tabla 5) (Figura 7).

La especie con mayor altura fue: Caracolí (Anacardium excelsum) con altura ente 28 a 34 $\mathrm{m}$, aunque con pocos individuos que constituyen con 2,2\%. Las especies con menor altura de $6 \mathrm{~m}$ fueron: Coquito (Adelia triloba), Caucho (Castilla elástica), Moral Bobo (Clarisia racemosa), Piñuelo (Duguetia peruviana), Chala (Erythrina sp), Bantano (Pithecellobium macradenium), Guayabo de monte (Psidium guajava L), Jaboncillo (Sapindus saponaria $L$ ), Tillo prieto (Sorocea sarcocarpa), con un mayor número de individuos en un porcentaje de $68,1 \%$ (Tabla 6) (Figura 8).

La especie con mayor frecuencia en ambos cuadrantes (A1 y A2) fue tillo prieto (Sorocea sarcocarpa), en

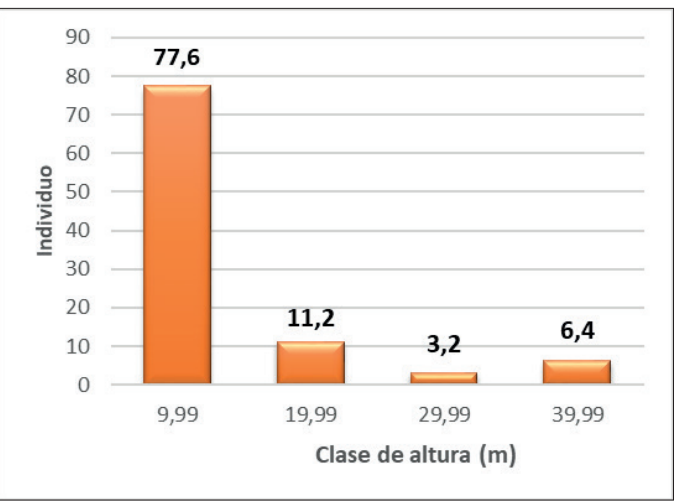

Figura 6. Representación de árboles por clase altura del cuadrante $\mathrm{A} 1$ en el bosque nativo de la Estación Pedro Franco Dávila. 
Tabla 4. Especies arbóreas presentes en el A2 en el bosque nativo de la Estación Pedro Franco Dávila.

\begin{tabular}{|c|c|c|c|c|}
\hline Nombre científico & Familia & Nombre común & $\begin{array}{c}\mathrm{N} .^{\circ} \text { de } \\
\text { individuo }\end{array}$ & $\%$ \\
\hline Sorocea sarcocarpa & Moraceae & Tillo prieto & 20 & 14,8 \\
\hline Erythrina $s p$ & Fabaceae & Chala & 8 & 5,9 \\
\hline Anacardium excelsum & Anacardiaceae & Caracolí & 16 & 11,8 \\
\hline Pithecellobium macradenium & Fabaceae & Bantano & 15 & 11,1 \\
\hline Duguetia peruviana & Annonaceae & Piñuelo & 29 & 21,4 \\
\hline Psidium guajava $L$. & Myrtaceae & Guayabo de monte & 4 & 2,9 \\
\hline Sapindus saponaria $L$. & Sapindaceae & Jaboncillo & 8 & 5,9 \\
\hline Adelia triloba & Euphorbiaceae & Coquito & 8 & 5,9 \\
\hline Castilla elástica & Moraceae & Caucho & 15 & 11,1 \\
\hline Spondias mombin $L$. & Anacardiaceae & Jobo & 1 & 0,7 \\
\hline Triplaris cumingiana & Polygonaceae & Fernán sanchez & 6 & 4,4 \\
\hline Clarisia racemosa & Moraceae & Moral Bobo & 5 & 3,7 \\
\hline
\end{tabular}

A1 tuvo 0,208 y A2 con 0,148; y las especies con menor frecuencia fueron: Fernán sanchez (Triplaris cumingiana), en el cuadrante A1 con 0,016 y Jobo (Spondias mombin L.) Con 0,007 en el cuadrante A2 (Tabla 7 y 8) (Figura 9).

Todas las especies se registraron en los dos cuadrantes a excepción de Tachuelo (Zanthoxylum aff. Panamense) que se registró solo en el cuadrante A1 y en el cuadrante A2 se registró solo la especie de Jobo (Spondias mombin L.). En ambos cuadrantes la familia más representativa fue Moraceae.

Cantidad de carbono que captura la especie arbórea más representativa del sistema arbolado nativo.

La especie Tillo prieto (Sorocea sarcocarpa) tiene mayor presencia en ambos cuadrantes del bosque nativo estación Pedro Franco Dávila; al azar se escogió un individuo de esta especie dentro del cuadrante tanto en el A1 y A2, para ser apeado y poder tomar las muestras que fueron enviadas al laboratorio para obtener carbono (c) mediante el método de análisis elemental, dando los siguientes resultados (Figura 10).

Tabla 5. Número de árboles por clase diamétrica del cuadrante A2 en el bosque nativo de la Estación Pedro Franco Dávila.

\begin{tabular}{ccc}
\hline Clase diamétrica & $\mathrm{N}^{\circ}{ }^{\circ}$ de individuo & $\%$ \\
\hline $0-6,99$ & 63 & 46,6 \\
$7-13,99$ & 40 & 29,6 \\
$14-20,99$ & 12 & 8,8 \\
$21-27,99$ & 8 & 5,9 \\
$28-34,99$ & 7 & 5,1 \\
$35-41,99$ & 3 & 2,2 \\
$42-48,99$ & 2 & 1,4 \\
\hline Total & 135 & 100 \\
\hline
\end{tabular}

\section{Cálculo de carbono}

El carbono total para el cuadrante A1 Tillo prieto (Sorocea sarcocarpa), con 26 individuos es de 23,51 $\mathrm{T}(\mathrm{c})$; con 7,53 T(c) para tronco; para copa 15,88 T(c); para ramas 0,054 T(c); para raíz 0,053 T(c) (Tabla 9).

El carbono total para el cuadrante A2 Tillo prieto (Sorocea sarcocarpa), con 20 individuos es de 7,58 $\mathrm{T}(\mathrm{c})$, con 5,62 T(c) para el tronco; con 1,32 T(c) para la copa; con 0,56 T(c) para las ramas; con 0,08 T(c) para la raíz (Tabla 10).

Dando un total de carbono para Tillo prieto (Sorocea sarcocarpa) en $5000 \mathrm{~m}^{2}$ en el área muestral de 31,09 $\mathrm{T}(\mathrm{c})$.

\section{Biomasa}

La biomasa viva constituye el fuste y copa del Tillo prieto (Sorocea sarcocarpa) en toneladas correspondiente 0,89 T(c), lo cual en el cuadrante A1 para 26 individuos da un total de $23,36 \mathrm{~T}$ (c) y el cuadrante A2 para 20 individuo un total de 6,81 T(c); con un total para el área estudiada de $30,17 \mathrm{~T}$ (c)

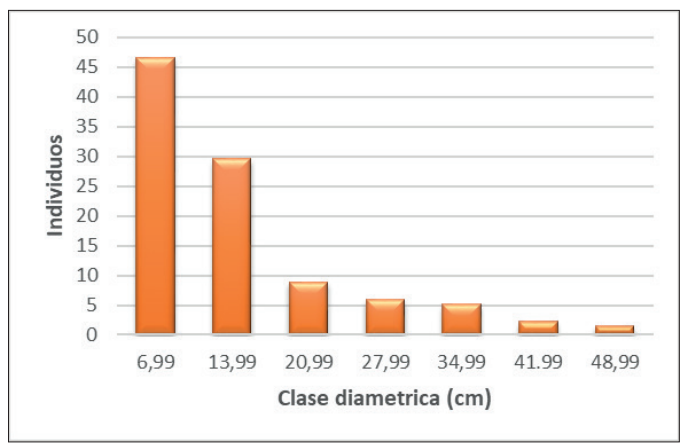

Figura 7. Representación de árboles por clase diamétrica del cuadrante $\mathrm{A} 2$ en el bosque nativo de la Estación Pedro Franco Dávila. 
Rev. Cient. Cien. Nat. Ambien. 15(1):245-257 Junio 2021
Mero \& Bonifaz • Sistema arbolado nativo y carbono en el Cantón Palenque, Provincia de los Ríos, Ecuador
Tabla 6. Número de árboles por clase altura del cuadrante A2 en el bosque nativo de la Estación Pedro Franco Dávila.

\begin{tabular}{ccc}
\hline $\begin{array}{c}\text { Clase } \\
\text { altura }\end{array}$ & $\begin{array}{c}\text { N. }{ }^{\circ} \text { de } \\
\text { individuo }\end{array}$ & $\%$ \\
\hline $0-6,99$ & 92 & 68,1 \\
$7-13,99$ & 28 & 20,7 \\
$14-20,99$ & 8 & 5,9 \\
$21-27,99$ & 4 & 2,9 \\
$28-34,99$ & 3 & 2,2 \\
\hline Total & 135 & 100 \\
\hline
\end{tabular}

Tabla 7. Especies del cuadrante A1 con mayor frecuencia en el bosque de la Estación Pedro Franco Dávila.

\begin{tabular}{|c|c|c|c|}
\hline Especies & $\begin{array}{c}\mathrm{N} .^{\circ} \text { de } \\
\text { individuos }\end{array}$ & $\%$ & $\mathrm{fr}$ \\
\hline Adelia triloba & 15 & 12 & 0,12 \\
\hline Anacardium excelsum & 3 & 2,4 & 0,024 \\
\hline Castilla elástica & 8 & 6,4 & 0,064 \\
\hline Clarisia racemosa & 5 & 4 & 0,04 \\
\hline Duguetia peruviana & 14 & 11,2 & 0,112 \\
\hline Erythrina sp. & 19 & 15,2 & 0,152 \\
\hline $\begin{array}{l}\text { Pithecellobium } \\
\text { macradenium }\end{array}$ & 19 & 15,2 & 0,152 \\
\hline Psidium guajava $L$ & 8 & 6,4 & 0,064 \\
\hline Sapindus saponaria L. & 3 & 2,4 & 0,024 \\
\hline Sorocea sarcocarpa & 26 & 20,8 & 0,208 \\
\hline Triplaris cumingiana & 2 & 1,6 & 0,016 \\
\hline $\begin{array}{l}\text { Zanthoxylum aff. } \\
\text { Panamense }\end{array}$ & 3 & 2,4 & 0,024 \\
\hline Spondias mombin L. & 0 & 0 & 0 \\
\hline Total & 125 & 100 & 1 \\
\hline
\end{tabular}

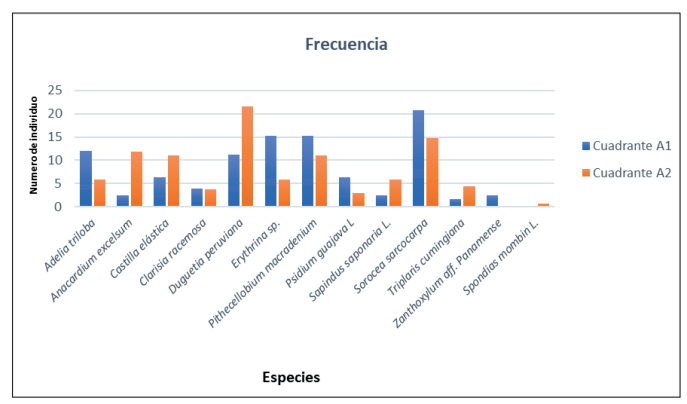

Figura 9. Representación de especies del cuadrante A1 y A2 con mayor frecuencia en el bosque de la Estación Pedro Franco Dávila.

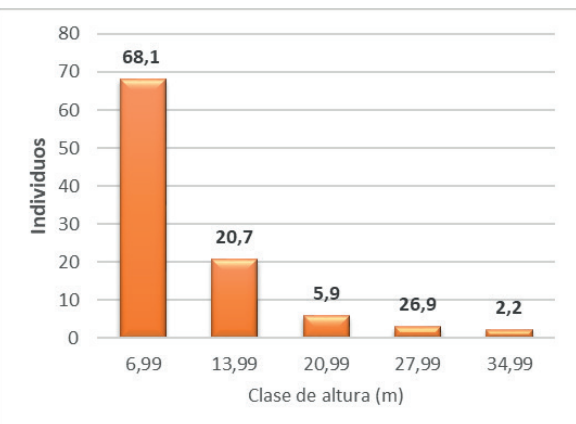

Figura 8. Representación de árboles por clase altura del cuadrante $\mathrm{A} 2$ en el bosque nativo de la Estación Pedro Franco Dávila.

Tabla 8. Especies del cuadrante A2 con mayor frecuencia en el bosque de la Estación Pedro Franco Dávila.

\begin{tabular}{|c|c|c|c|}
\hline Especies & $\begin{array}{c}\mathrm{N} .^{\circ} \text { de } \\
\text { individuos }\end{array}$ & $\%$ & $\mathrm{fr}$ \\
\hline Adelia triloba & 8 & 5,9 & 0,059 \\
\hline $\begin{array}{l}\text { Anacardium } \\
\text { excelsum }\end{array}$ & 16 & 11,8 & 0,118 \\
\hline Castilla elástica & 15 & 11,1 & 0,11 \\
\hline Clarisia racemosa & 5 & 3,7 & 0,037 \\
\hline $\begin{array}{l}\text { Duguetia } \\
\text { peruviana }\end{array}$ & 29 & 21,4 & 0,21 \\
\hline Erythrina sp. & 8 & 5,9 & 0,059 \\
\hline $\begin{array}{l}\text { Pithecellobium } \\
\text { macradenium }\end{array}$ & 15 & 11,1 & 0,11 \\
\hline Psidium guajava $L$ & 4 & 2,9 & 0,029 \\
\hline $\begin{array}{l}\text { Sapindus } \\
\text { saponaria } L .\end{array}$ & 8 & 5,9 & 0,059 \\
\hline $\begin{array}{l}\text { Sorocea } \\
\text { sarcocarpa }\end{array}$ & 20 & 14,8 & 0,14 \\
\hline $\begin{array}{l}\text { Triplaris } \\
\text { cumingiana }\end{array}$ & 6 & 4,4 & 0,04 \\
\hline $\begin{array}{l}\text { Zanthoxylum aff. } \\
\text { Panamense }\end{array}$ & 0 & 0 & 0 \\
\hline $\begin{array}{l}\text { Spondias mombin } \\
\text { L. }\end{array}$ & 1 & 0,740 & 0,0074 \\
\hline Total & 135 & 100 & 1 \\
\hline
\end{tabular}




\begin{tabular}{|c|c|c|c|c|}
\hline \multirow{3}{*}{0 를 } & \multirow{4}{*}{$\begin{array}{l}\text { DIRECCION DE } \\
\text { INVESTIGACIÓN } \\
\text { Y VINCULACIÓN }\end{array}$} & \multirow{2}{*}{ 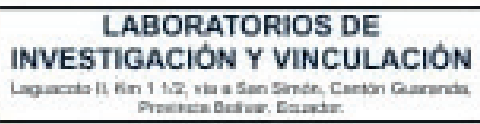 } & Codiligo & EPG12-02 \\
\hline & & & Versión & 1 \\
\hline & & \multirow{2}{*}{ INFORME DE RESULTADOS } & Año & 2021 \\
\hline Extrats & & & Pagina & Pagina 1 de 1 \\
\hline
\end{tabular}

INFORME DE ENSAYOS N ${ }^{\circ}$ INV 003

\begin{tabular}{|c|c|c|c|c|c|}
\hline \multicolumn{6}{|c|}{ DESCRIPCIÓN DE LA MUESTRA } \\
\hline \multicolumn{2}{|l|}{ Solicitanto } & \multicolumn{4}{|c|}{ Srta. Patricia Lorena Mero Lucas } \\
\hline \multicolumn{2}{|l|}{ Muestra } & \multicolumn{4}{|c|}{ Tejido vegetal } \\
\hline \multicolumn{2}{|c|}{ Código asignado UEB } & \multicolumn{4}{|c|}{ INV 10- INV 11- INV 12- INV 13-INV 14} \\
\hline \multicolumn{2}{|c|}{ Estado de la muestra } & \multicolumn{4}{|c|}{ Sólido } \\
\hline \multicolumn{2}{|c|}{ Envase de recepción } & \multicolumn{4}{|c|}{ Frascos de plástico } \\
\hline \multicolumn{2}{|c|}{ Análisis requerido(s) } & \multicolumn{4}{|c|}{ Porcentajo de Carbono } \\
\hline \multicolumn{2}{|c|}{ Fecha de recepción } & \multicolumn{4}{|c|}{$05 / 02 / 2021$} \\
\hline \multicolumn{2}{|c|}{ Focha do análisis } & \multicolumn{4}{|l|}{$11 / 02 / 20201$} \\
\hline \multicolumn{2}{|c|}{ Fecha de informe } & \multicolumn{4}{|l|}{$12 / 02 / 2021$} \\
\hline \multicolumn{2}{|c|}{ Técnico (s) asignado } & \multicolumn{4}{|l|}{ MIPV } \\
\hline \multicolumn{6}{|c|}{ RESULTADOS OBTENIDOS } \\
\hline $\begin{array}{l}\text { Código de } \\
\text { laboratorio }\end{array}$ & Muestra & Parámetro & Unidad & Método & $\begin{array}{c}\text { Resultado } \\
\text { (\%) }\end{array}$ \\
\hline INV 10 & $P m-R$ & Carbon en base seca & $\%$ & $\begin{array}{l}\text { Análisis Elemental } \\
\text { UNE_EN } 15104\end{array}$ & 48.97 \\
\hline INV 11 & Pm-Tr & Carbon en base seca & $\%$ & $\begin{array}{l}\text { Análisis Elemental } \\
\text { UNE_EN } 15104\end{array}$ & 45.95 \\
\hline INV 12 & $\mathrm{Pm}-\mathrm{Rm}$ & Carban en base seca & $\%$ & $\begin{array}{l}\text { Análisis Elemental } \\
\text { UNE_EN } 15104\end{array}$ & 50.01 \\
\hline INV 13 & $\mathrm{Pm}-\mathrm{H}$ & Carban en base seca & $\%$ & $\begin{array}{l}\text { Análisis Elemental } \\
\text { UNE_EN } 15104\end{array}$ & 46.67 \\
\hline INV 14 & $\mathrm{Pm}-\mathrm{Hr}$ & Carbon en base seca & $\%$ & $\begin{array}{l}\text { Análisis Elerrental } \\
\text { UNE_EN } 15104\end{array}$ & 39.18 \\
\hline
\end{tabular}

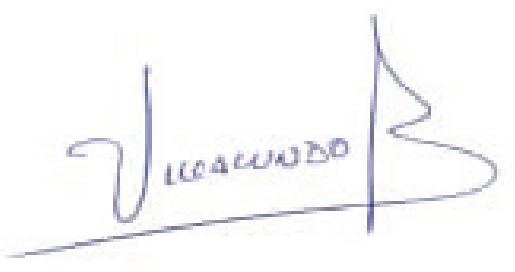

Ing. Marcelo Vilcacundo

Director DIVIUEB

Figura 10. Resultados del laboratorio de análisis elemental de las muestras de raíz, tronco, hoja, rama y hojarasca de (Sorocea sarcocarpa) Tillo prieto. 
Rev. Cient. Cien. Nat. Ambien. 15(1):245-257 Junio 2021
Mero \& Bonifaz - Sistema arbolado nativo y carbono en el Cantón Palenque, Provincia de los Ríos, Ecuador

Tabla 9. Carbono de tronco, copa, rama y raíz en árboles de tillo prieto de la estación Pedro Franco Dávila del Cuadrante A1.

\begin{tabular}{|c|c|c|c|c|}
\hline Muestra & $\begin{array}{c}\text { Clase de } \\
\text { altura }\end{array}$ & $\begin{array}{c}\mathrm{N} .^{\circ} \\
\text { Individuo }\end{array}$ & Resultado (\%) & $\begin{array}{c}\text { Resultado } \\
\text { (T) }\end{array}$ \\
\hline Tronco & $0-6,99$ & 9 & 45,95 & 1,98 \\
\hline Tronco & 7-13.99 & 11 & 45,95 & 3,74 \\
\hline Tronco & $14-20,99$ & 1 & 45,95 & 0,29 \\
\hline Tronco & $21-27,99$ & 1 & 45,95 & 0,24 \\
\hline Tronco & $28-34,99$ & 4 & 45,95 & 1,28 \\
\hline Copa & $0-6,99$ & 9 & 45,95 & 0,86 \\
\hline Copa & $7-13.99$ & 11 & 45,95 & 1,1 \\
\hline Copa & $14-20,99$ & 1 & 45,95 & 0,12 \\
\hline Copa & $21-27,99$ & 1 & 45,95 & 2,6 \\
\hline Copa & $28-34,99$ & 4 & 45,95 & 11,2 \\
\hline Rama & $0-6,99$ & 9 & 50,01 & 0,0315 \\
\hline Rama & 7-13.99 & 11 & 50,01 & 0,0187 \\
\hline Rama & $14-20,99$ & 1 & 50,01 & 0,0011 \\
\hline Rama & $21-27,99$ & 1 & 50,01 & 0,0008 \\
\hline Rama & $28-34,99$ & 4 & 50,01 & 0,0028 \\
\hline Raíz & $0-6,99$ & 9 & 48,97 & 0,0306 \\
\hline Raíz & $7-13.99$ & 11 & 48,97 & 0,0187 \\
\hline Raíz & $14-20,99$ & 1 & 48,97 & 0,0011 \\
\hline Raíz & $21-27,99$ & 1 & 48,97 & 0,00083 \\
\hline Raíz & $28-34,99$ & 4 & 48,97 & 0,00272 \\
\hline TOTAL & & & & 23,51 \\
\hline
\end{tabular}


Tabla 10. Carbono de tronco, copa, rama y raíz en árboles de tillo prieto de la Estación Pedro Franco Dávila del Cuadrante A2.

\begin{tabular}{|c|c|c|c|c|}
\hline Muestra & $\begin{array}{c}\text { clase } \\
\text { de altura }\end{array}$ & $\begin{array}{c}\mathrm{N} \cdot{ }^{\circ} \\
\text { Individuo }\end{array}$ & $\begin{array}{c}\text { Resultado } \\
\text { (\%) }\end{array}$ & $\begin{array}{l}\text { Resultado } \\
\text { (T) }\end{array}$ \\
\hline Tronco & $0-2,99$ & 5 & 45,95 & 1,1 \\
\hline Tronco & $3-5,99$ & 7 & 45,95 & 2,1 \\
\hline Tronco & $6-8,99$ & 6 & 45,95 & 1,68 \\
\hline Tronco & $9-11,99$ & 1 & 45,95 & 0,36 \\
\hline Tronco & $12-14,99$ & 1 & 45,95 & 0,38 \\
\hline Copa & $0-2,99$ & 5 & 45,95 & 0,6 \\
\hline Copa & $3-5,99$ & 7 & 45,95 & 0,28 \\
\hline Copa & $6-8,99$ & 6 & 45,95 & 0,36 \\
\hline Copa & $9-11,99$ & 1 & 45,95 & 0,05 \\
\hline Copa & $12-14,99$ & 1 & 45,95 & 0,02 \\
\hline Rama & $0-2,99$ & 5 & 50,01 & 0,0415 \\
\hline Rama & $3-5,99$ & 7 & 50,01 & 3,5007 \\
\hline Rama & $6-8,99$ & 6 & 50,01 & 0,0162 \\
\hline Rama & $9-11,99$ & 1 & 50,01 & 0,0020 \\
\hline Rama & $12-14,99$ & 1 & 50,01 & 0,0016 \\
\hline Raíz & $0-2,99$ & 5 & 48,97 & 0,0405 \\
\hline Raíz & $3-5,99$ & 7 & 48,97 & 0,028 \\
\hline Raíz & $6-8,99$ & 6 & 48,97 & 0,0156 \\
\hline Raíz & $9-11,99$ & 1 & 48,97 & 0,0018 \\
\hline Raíz & $12-14,99$ & 1 & 48,97 & 0,0020 \\
\hline TOTAL & & & & 7,58 \\
\hline
\end{tabular}




\section{Volumen de ramas.}

El volumen de ramas mediante los datos que se tomó en el campo del tillo prieto (Sorocea sarcocarpa) donde se contó 15 ramas y una longitud total fue de $2.8 \mathrm{~m}$ y el diámetro de rama es de $1.5 \mathrm{~cm}$ dando como resultado:

$$
\begin{gathered}
\operatorname{Vr}=(\pi)(r 2)(h) \\
V r=(3.1416)(0,56)(2,08)=3.65 \mathrm{~m}^{3} .
\end{gathered}
$$

Volumen comercial

Para el volumen comercial se tomó la medida del fuste del Tillo prieto (Sorocea sarcocarpa) que fue de $15 \mathrm{~m}$ de altura comercial y diámetro $53 \mathrm{~cm}$ dando como resultado:

$$
\mathrm{VC}=\mathrm{H} . \mathrm{AB} \quad A B=\frac{\mathrm{D} \cdot \mathrm{H}}{\text { Area }}
$$

El volumen comercial de un tillo prieto es de $4.8 \mathrm{~m}^{3}$.

Cuadrante A1 $(26)=124.8 m$ Cuadrante A2 $(20)=96 \mathrm{~m}^{3}$

\section{Volumen de raíces}

Para el volumen de raíces los datos obtenidos del campo que se tomó del Tillo prieto (Sorocea sarcocarpa) se contaron 15 raíces, diámetro de la raíz fue $1.0 \mathrm{~cm}$ y la longitud total fue $2,08 \mathrm{~m}$ dando como resultado:

$$
\begin{gathered}
. V r=(\pi)(r 2)(h) \\
V r=(3.1416)(0,25)(2,08)=1.63 \mathrm{~m}^{3}
\end{gathered}
$$

\section{Hojarasca}

La muestra de referencia 10 gramos de hojarasca que se envió analizar por el método de análisis elemental dio 39,18 \% (c) que seria 3,92 T (c) por lo cual los 680 gramo de hojarasca recogido en el área de estudio daría 266,6 T (c).

\section{Hojas}

La muestra de 10 gramos de hojas vivas que se envió analizar por el método de análisis elemental dio 46,67 $\%$ (c) que seria 4,67 T (c). Por lo cual la muestra de los 1359 gramos de hojas tomada en el área de estudio daría como resultados 634,6 T (c).

\section{Discusión}

En Ecuador para el almacenamiento de carbono, se han realizados trabajos en distintas Provincias lo cual, Castillo (2014) registro 776 individuos con 56 especies donde se estimó el carbono total almacenado de raíz, tallo, ramas y hojas de las especies más abundante que fueron Fernán sanchez (Triplaris cumingiana) con 29.14 t/ha, Pacay blanco (Cupania cinérea) con 30.45 t/ha, Laurel (Cordia alliodora) con 19.95 t/ha, Tillo, clavo (Pseudolmedia rigida) con $6.31 \mathrm{t} / \mathrm{ha}$, Membrillo (Inga oerstediana) con 18.85 t/ha, Guaba de mono (Eschweilera integrifolia) con $20.57 \mathrm{t} / \mathrm{ha}$, Vara blanca (Allophylus psilospermus) con $4.8 \mathrm{t} / \mathrm{ha}$. El presente estudio registro 255 individuo con 13 especies de las cuales la más representativa fue Tillo prieto (Sorocea sarcocarpa) con 4.8 T de carbono.
Arevalo (2015), Ramirez \& Zamora (2019) utilizaron el método indirecto para el medir el almacenamiento de carbono en biomasa aérea, en el bosque natural Tinajillas-Limon Indanza y el Bosque Nativo de la Comunidad Rancho Chico, la cantidad de carbono reportado fue de 0,064 ton y 1011.18 ton para cada sitio antes indicado los datos reportados difieren, aunque dichos autores utilizaron el mismo método.

Bajaña (2016) evaluó el almacenamiento de carbono en el Bosque Protector Pedro Franco Dávila, en un área natural y un área intervenida, reportando que la especie con mayor almacenamiento de carbono en un área natural fue Tutumbe (Enterolobium cyclocarpum) con 50,066 kg ha-1 y en área intervenida fue Fernán Sánchez (Triplaris cumingiana) con 29,219 $\mathrm{kg}$ ha-1. El presente estudio se realizó dentro de unas áreas intervenida del mismo Bosque Protector siendo el Tillo prieto (Sorocea sarcocarpa) la especie más representativa de este tipo de área con un almacenamiento de carbono de 4,8 T (c); mientras que Fernán Sánchez (Triplaris cumingiana) tuvo menor presencia en el área estudiada. Coincidiendo ambos estudios que la familia Morácea es la más representativa de ambos sectores,

\section{Conclusiones}

Tillo prieto (Sorocea sarcocarpa) es la especie más representativa del área de estudio, por tener un mayor número de individuos en cada cuadrante, sin llegar a ser dominante en el área muestral del bosque nativo.

La especie que presentó mayor abundancia en el área estudiada fue (Sorocea sarcocarpa) Tillo prieto con 46 individuos, mientras que la especie con menor abundancia fue (Triplaris cumingiana) Fernán Sánchez con 8 individuos en el área estudiada.

Tillo prieto (Sorocea sarcocarpa) en el área estudiada tiene un índice de captura de carbono en tronco 13,15 $\mathrm{T}(\mathrm{c})$; copa 17,2 $\mathrm{T}(\mathrm{c})$; rama $0,61 \mathrm{~T}(\mathrm{c})$; raíz $0,13 \mathrm{~T}(\mathrm{c})$, siendo su volumen comercial de $4.8 \mathrm{~m}^{3}$.

La población de Tillo prieto (Sorocea sarcocarpa), es joven debido a que sus individuos se encuentran el cuadrante A1 en clase de altura entre 7-13,99 y en el cuadrante $A 2$ en clase de altura entre 6- 8,99.

\section{Recomendaciones}

- Fomentar estudios en la Estación Científica Pedro Franco Dávila con especies nativas para tener un registro de la cantidad de carbono que almacenan las diferentes especies nativas, a fin de comprender el rol de los árboles nativos.

- Realizar investigaciones de almacenamiento de carbono en la especie adulta del Tillo prieto (Sorocea sarcocarpa) para comparar los datos con una población juvenil. 


\section{Referencias}

Arevalo, C. (2015). Medicion de carbono del estracto arboreo en un area del bosque natural Tinajillas- Limon Indanza. Cuenca.

Bajaña, S. (2016). ESTRUCTURA VEGETAL

DEL BOSQUE Y SU CONTRIBUCIÓN

A LA CAPTACIÓN DE CARBONO EN

LA RESERVA PEDRO FRANCO DAVILA

JAUNECHE-ECUADOR. AÑO 2015.

PROPUESTA DE OFERTA DE CARBONO.

. Obtenido de https://repositorio. uteq.edu.ec/handle/43000/1667/ browse?type=author\&order $=$.

Brown, S., \& Lugo, A. (1984). Biomass and CarbonContent of Saplings in Puert Rican Secondary Forests. . Caribbean Journal of Science. 36(3): 346-350.

Castillo, R. (2014). Estimacion del contenido de carbono en las especies arboreas en el predio "El Prado" del sitioremolino para la conservacion de bosques del canton Santa Rosa .

FAO. (2006). Organizacion de las Naciones Unidas para la Alimentacion y la Agricultura. Los bosque y el cambio climatico. La gestionb forestal es funbdamenbtal para afronbtar el camnbio climatico. Disponible http://WWW.fao.org/newsroom/es/ focus/2006/1000247/in.html.dex.

Fernandez , I., Delgado, M., Bellet, M., \& Garcia, E. (2011). BOSQUE DEL MUNDO II. Real Jardin Botanico, CSIC.

Francis, J. (2000). Estimating Biomass and Carbon Content of Saplings in Puerto Rican Secondary Forests. . Caribbean Journal of Science. 36(3): 346-350.

Gifford, R. (2000). Carbon contens of above-ground tissues of forest and woodland tress. Australian Greenhouse Office: National Carbon Accounting Systema. Technical Report N ${ }^{\circ} 22$. Canberra.17 pp.

Google Earth. (2018). Google Earth. Obtenido de Recuperado el 23 de febrero de 2021, de https: / / earth.google.com/web

IPCC. (1996). Mexico citty: Intergovernmental Panel on Climate Change. Report of the twelfh session of the intergovernmental panel on climate change. Reference manual and workbook of the IPCC 1996 revised guidelines for national greenhouse gas inventories. .

IPCC. (2000). Intergovernmental Panel on Climate Change. IPCC Special Report: Land Use, Land Use Change and Forestry. Cambridge University Press.

IPPCC. (2007). (Intergovernmental Panel on Climate Change). 2007. Climate Change 2007: The Physical Science Basis. Summary for Policymakers. Paris, Francia. s.e. p 2.

Lee Pinto, G. (2002). Estudio Preliminar para la Estimación de Biomasa y Cuantificación de Carbono para Vochysia guatemalensis,
Calophyullum brasilense y Cybistax donnellsmithii en bosques de Guatemala. . Universidad de San Carlos de Guatemala.: Facultad de Agronomía. Instituto de Investigaciones Agronomicas. Guatemala.

Martel, C. (2012). ). Cuantificación del Carbono almacenado en formaciones vegetales amazónicas en CICRA", Madre de Dios . Peru.

Ramirez, L., \& Zamora, W. (2019). Evaluacion de la biomasa area y carbono fijo en el bosque nativo de la comunidad Rancho Chico, Sector Cochapamba-Imbabura.

Rugnitz, M., Chacon, M., \& Porro, R. (2009). Guia para la determinacion de carbono en pequeñas Propiedades Rurales. Lima, Peru: Centro Mundial Agroflorestal (ICRAF) / Consorcio Iniciativa Amazonica (IA) .

Soares, C., \& Oliveira, M. (2000). Equacoes para estimar a quantidade de carbono parte area de arboles de eucalipto. Minas Gerais: Arvore 26 (5):533-539.

Veracruzana. (2009). Evaluacion del Co2 almacenado en la vegetacion del bosque nativo de Santigo Del Estero (Argentina): Bases para la conservacion de bosque en regeneracion. Foresta Veracruzana, vol. 11, num.2, 2009,pp. 1-10. 